\title{
Index to Volume 132
}

\section{Compiled by William Halliday}

Activity, 20

Adam, C.I.G., 319

Aeronautes saxatalis, 386

Agaricomycetes, 407

Alaska, North Slope, 268, 382

Alberta,

Southeastern, 140

Southwestern, 168

Alvar, 238

Ambystoma macrodactylum, 168

Amphibian, 43, 46, 53, 58, 61, 163, 168, 176, 223

Anaxyrus

boreas, 53

fowleri, 46

Angoh, S.Y.J., 122

Apalone spinifera, 120

Arachnida, 330

Araneae, 330

Arctic, 254, 279

Arsenault, M.A., 330

Asphalt, 103

Assessment, Species Status, 176

Atkinson-Adams, M.R., C.J. Price, G.J. Scrimgeour, C.A. Paszkowski. Long-toed Salamander ( $\mathrm{Am}$ bystoma macrodactylum) hibernacula in Waterton Lakes National Park revealed using Passive

Award, Integrated Transponder Telemetry, 168-175

James Fletcher, 85

Order of Canada, 86

Azolla cristata, 350

Basidiomycetes, 407

Basidiomycota, 407

Bathyraja lindbergi, 261

Batrachochytrium dendrobatidis, 53

Behaviour, Caching, 285

Bennett, R., 330

Bickerton, H.J., 350

BioBlitz, 394

Biodiversity, 394

Native, 350

Biogeography, 394

Biology, Winter, 61

Bird, 211, 254, 279, 285

Bishop, C.A., 30

Bison, 219

Bison bison, 219

Blagoev, G.A., 330

Blaney, S., 389

Blattodea, 319

Blouin-Demers, G., 25, 223
Body, Fruiting, 407

Boettgerilla pallens, 264

Bonds, Pair, 211

Bowden, J.J., K.M. Knysh, G.A. Blagoev, R. Bennett, M.A. Arsenault, C.F. Harding, R.W. Harding, R. Curley. The spiders of Prince Edward Island: experts and citizen scientists collaborate for faunistics, 330-349

Branta canadensis, 211

Breeding, 20, 254

British Columbia, 36

Northwestern, 53

Queen Charlotte Sound, 261

Southern, 30, 386

Brodo, I., 95

Brooks, R.J., 20

Bruce, M., T. Linnansaari, R.A. Currey. First record of Eurasian Water-milfoil, Myriophyllum spicatum, for the Saint John River, New Brunswick, 231237

Brunton, D.F. Distribution and taxonomy of Isoetes tuckermanii subsp. acadiensis, comb. nov. (Isoetaceae) in North America, 360-367

Brunton, D.F. Swimming as a potentially important emergency capability of White-throated Swifts (Aeroneautes saxatalis) engaged in aerial mating, 386-388

Brunton, D.F., H.J. Bickerton. New records for Eastern Mosquito Fern (Azolla cristana, Salviniaceae) in Canada, 350-359

Bullsnake, 126, 140

Bycatch, 61

Caches, Tree, 285

Caching, Food, 285

Cairns, N.A., P.L. Rutherford, D.J. Hoysak. Morphology, reproduction, habitat use, and hibernation of Red-bellied Snake (Storeria occipitomaculata) near its northern range limit, 150-162

Calving, 219

Capture, 20

Caribou, 382

Carstairs, S., M. Dupuis-Desormeaux, C.M. Davy. Revisiting the hypothesis of sex-biased turtle road mortality, 289-295

Catling, P., 319

Catling, P.M., B. Kostiuk. A Canadian range extension for Wormslug (Boettgerilla pallens; Gastropoda: Stylommatophora: Boettgerillidae), 264-267

Checklist, 176, 319

Chelydra serpentina, 4, 8, 103, 122, 289, 378

Chickadee, Black-capped, 368 
Choquette, J.D., E.A. Jolin. Checklist and status of the amphibians and reptiles of Essex County, Ontario: a 35 year update, $176-190$

Chrysemys

picta, 20, 108, 289

picta bellii, 108

Cipriani, J., 95

Clemmys guttata, 18

Climate Change, 279, 350

Climbing, 58

Colour Variation, 43

Colouration, 43

Coluber constrictor mormon, 30

Complex, Ojibway Prairie, 176

Condition, Body, 368

Connecticut, New Haven, 211

Conover, M.R., J.B. Dinkins. Divorce in Canada Geese (Branta canadensis): frequency, causes, and consequences, $211-218$

Conservation, 30, 168, 176, 394 Fungal, 407

Cooke, S.J., 61

Cool-climate, 150

County, Essex, 176

Cray, H.A., W.H. Pollard. Use of stabilized thaw slumps by Arctic birds and mammals: evidence from Herschel Island, Yukon, 279-284

Crotalus oreganus oreganus, 30

Crowell, M., 163

Curley, R., 330

Currey, R.A., 231

Davy, C.M., 122, 289

Davy, C.M., J. Skuza, A.K. Whitear. Spiny Softshell (Apalone spinifera) turtles exhibit scarring consistent with attempted lamprey bites, 120-121

De Solla, S.R., J.A. Gugelyk. Oviposition and subsequent depredation of Snapping Turtle (Chelydra serpentina) nests in fresh asphalt, 103-107

DeBruyn, A., 53

Depredation, 103, 122

Dermaptera, 319

Diet, 268

Dinkins, J.B., 211

Dispersal, Sex-biased, 289

Distribution, 4, 8, 264, 360 Size, 140

Disturbance, 279

Diversity, Diet, 268

Divorce, 211

Dolomite, 238

Dorendorf, R.R., K.J. Sivy, M.D. Robards, T.W. Glass, K.L. Pilgrim. Spring food habits of Wolverine (Gulo gulo) in the Colville River watershed, Alaska, 268-278

Dorval, H., 394

Doucet, D.A., 319

Drotos, K., 394

Dupuis-Desormeaux, M., 289
Ecology, 264

Nesting, 8

Road, 289

Spatial, 46, 108, 126

Urban, 108

Winter, 61

Ecosystem, 238

Alvar, 238

Endangered, 238

Editorial, 1, 99

Editors' Report for Volume 131 (2017), 316-318

Edkins, T.L., C.M. Somers, M.C. Vanderwel, M.J. Sadar, R.G. Poulin. Variable habitat selection and movement patterns among Bullsnake (Pituophis catenifer sayi) populations in Saskatchewan, 126-139

Elliott, K.H., 368

Emydoidea blandingii, 122, 289

Endemic, Acadian, 360

Erosion, Coastal, 279

Erythrism, 43

Exclusion Fencing, 30

Eye, D.M., J.R. Maida, O.M. McKibbin, K.W. Larsen, C.A. Bishop. Snake mortality and cover board effectiveness along exclusion fencing in British Columbia, Canada, 30-35

Farr, D.R., 36

Faunistics, 330

Fern, Eastern Mosquito, 350

Fidelity, Mate, 211

Fish, 261

Fitness, 25

Foraging, 58

Formica, 150

Frei, B., 368

Friesen, C., 238

Frog,

Fungi, 407

Northern Leopard, 223

Spring Peeper, 43, 163

Fungus, Amphibian Chytrid, 53

Galois, P., È.-L. Grenier, M. Ouellet. New size record for Snapping Turtle (Chelydra serpentina) in southern Quebec, Canada, 378-381

Gartersnake,

Common, 25, 223

Western, 36

Gastropod, 264

Geese, Canada, 211

Gilhen, J., 8, 43

Gilhen, J., T. Power. Snapping Turtle-Tortue serpentine - turtle mi' kjikj (snapping; Chelydra serpentina), added to the herpetofauna of Cape Breton Island, Nova Scotia, Canada, 4-7

Glass, T.W., 268

Gophersnake, Great Basin, 30

Graptemys geographica, 122, 289 
Green, D.M., K.T. Yagi. Ready for bed: pre-hibernation movements and habitat use by Fowler's Toads (Anaxyrus fowleri), 46-52

Gregory, P.T., D.R. Farr. Factors affecting litter size in Western Gartersnake (Thamnophis elegans) in British Columbia: place, time, and size of mother, 36-42

Grenier, Ė.-L., 378

Growth Rate, 25

Gugelyk, J.A., 103

Gulo gulo, 268, 382

Habitat, 25, 46, 58, 108, 126, 140, 150, 168, 223

Breeding, 53

Loss, 176

Terrestrial, 168

Habits, Food, 268

Halliday, W.D., 99

Halliday, W.D., G. Blouin-Demers. Body temperature influences growth rates of Common Gartersnakes (Thamnophis sirtalis), 25-29

Halliday, W.D., G. Blouin-Demers. Habitat selection by Common Gartersnakes (Thamnophis sirtalis) is affected by vegetation structure but not by location of Northern Leopard Frog (Lithobates pipiens) prey, 223-230

Halliday, W.D., J.M. Saarela. James Fletcher Award for The Canadian Field-Naturalist Volume 131, 85

Halliday, W.D., D.C. Seburn. Introduction to the Special Issue on herpetology in Canada, 1-3

Hamel, C., 238

Hanrahan, C., 95

Harding, C.F., 330

Harding, R.W., 319, 330

Hay, C.R.J., R.G. Thorn, C.R. Jacobs. Taxonomic survey of Agaricomycetes (Fungi: Basidiomycota) in Ontario tallgrass prairies determined by fruiting body and soil rDNA sampling, 407-424

Hendricks, P. Clark's Nutcrackers (Nucifraga columbiana) caching Whitebark Pine (Pinus albicaulis) seeds in trees, 285-288

Herpetofauna, 4, 8, 18, 20, 25, 30, 36, 43, 46, 53, 58, $61,103,108,120,122,126,140,150,163,168$, $176,223,289,378$

Hibernacula, 140, 150, 168

Hibernation, 46, 108, 140, 150, 168

Homing, 58

Hook, Circle, 61

Hoysak, D.J., 150

Hypericaceae, 389

Hypericum sphaerocarpum, 389

Ichthyostomyzon unicuspis, 120

iNaturalist, 434

Index,

Condition, 368

Scaled Mass, 368

Interaction, Human, 140

Introduced, 264
Invasive,

Aquatic, 231

Species, 231, 264

Ireland, D., 394

Island,

Cape Breton, 4, 8

Herschel, 279

Pelee, 176

Isoetes

acadiensis, 360

tuckermanii, 360

tuckermanii subsp. acadiensis, 360

Jacobs, C.R., 407

Jolin, E.A., 176

Jung, T.S., N.C. Larter, T. Powell. Early and late births in high-latitude populations of free-ranging Bison (Bison bison), 219-222

Karson, A., S.Y.J. Angoh, C.M. Davy. Depredation of gravid freshwater turtles by Raccoons (Procyon lotor), 122-125

Karst, 238

Keech, M.A., 382

King, J.R., G.A. MacFarlane, T.B. Zubkowski. First records of Commander Skate (Bathyraja lindbergi) in Canadian Pacific waters, 261-263

Klymko, J., P. Catling, J.B. Ogden, R.W. Harding, D.F. McAlpine, S.L. Robinson, D.A. Doucet, C.I.G. Adam. Orthoptera and allies in the Maritime provinces, Canada: new records and updated provincial checklists, 319-329

Knysh, K.M., 330

Kostiuk, B., 264

Labrador, 163

Laird, C.R., 382

Lake,

Erie, 120

Nipissing, 61

Lamont, M.M. New avian breeding records for Kugluktuk, Nunavut, 254-260

Lamprey,

Parasitic, 120

Sea, 120

Silver, 120

Larsen, K.W., 30

Larter, N.C., 219

LeGros, D.L. The use of an anthropogenic structure by Eastern Red-backed Salamander (Plethodon cinereus), 58-60

Lennox, R.J., W.M. Twardek, S.J. Cooke. Observations of Mudpuppy (Necturus maculosus) bycatch in a recreational ice fishery in northern Ontario, 61-66

Lepitzki, D., A. Martin. Editors' Report for Volume 131 (2017), 316-318

Letharia columbiana, 285

Lichen, 394

American Wolf, 285 
Limestone, 238

Linnansaari, T., 231

Lithobates pipiens, 223

Litzgus, J.D., 20

Longevity, 18

Lycophyte, 360

MacFarlane, G.A., 261

Magoun, A.J., C.R. Laird, M.A. Keech, P. Valkenburg, L.S. Parrett, M.D. Robards. Predation on Caribou (Rangifer tarandus) by Wolverine (Gulo gulo) after long pursuits, 382-385

Maida, J.R., 30

Mammal, 219, 268, 279

Management, Fisheries, 61

Manitoba, Interlake, 238

Southwestern, 150

Mantodea, 319

Marchand, K.A., C.M. Somers, R.G. Poulin. Spatial ecology and multi-scale habitat selection by Western Painted Turtles (Chrysemys picta bellii) in an urban area, 108-119

Marine, 261

Maritime, 43, 319, 330

Martin, A., 316

Martin, A.E. Francis Cook Appointed to the Order of Canada for his Exceptional Contributions to Canadian Herpetology and The Canadian FieldNaturalist, 86

Martin, A.E. iNaturalist Canada passes the 1000000 observation mark, 434

Mass, Body, 368

Mating, 20 Aerial, 386

McAlpine, D.F., 319

McAlpine, D.F., J. Gilhen. Erythrism in Spring Peeper (Pseudacris crucifer) in Maritime Canada, 4345

McKibbin, O.M., 30

McLachlan Hamilton, K., 95

McMullin, R.T., K. Drotos, D. Ireland, H. Dorval. Diversity and conservation status of lichens and allied fungi in the Greater Toronto Area: results from four years of the Ontario BioBlitz, 394406

Minutes of the $139^{\text {th }}$ Annual Business Meeting (ABM) of the Ottawa Field-Naturalists' Club 9 January $2018,87-88$

Mitigation, 30

Moldowan, P.D., R.J. Brooks, J.D. Litzgus. Sex-biased seasonal capture rates in Painted Turtle (Chrysemys picta), 20-24

Monogamy, 211

Montana, 285

Morphology, 150

Mortality, 30

Additive, 122

Road, 289

Movement, 46
Mudpuppy, 61

Mushrooms, 407

Mycota, Grassland, 407

Myriophyllum spicatum, 231

Necturus maculosus, 61

Neufeld, R., C. Hamel, C. Friesen. Manitoba's endangered alvars: an initial description of their extent and status, 238-253

New Brunswick, 43 Fredericton, 231

Nip, E.J., B. Frei, K.H. Elliott. Seasonal and temporal variation in scaled mass index of Black-capped Chickadees (Poecile atricapillus), 368-377

North America, 360

Nova Scotia, 4, 8, 43

Nucifraga columbiana, 285

Nunavut, Kugluktuk, 254

Nutcracker, Clark's, 285

Ogden, J.B., 319

Oldham, M.J., W.D. Van Hemessen, S. Blaney. Roundfruited St. John's-wort (Hypericum sphaerocarpum, Hypericaceae) in Canada, 389-393

Ontario,

Central, 20, 58, 103, 289

Eastern, 18, 223

Frontenac Axis, 350

Greater Toronto Area, 394

Long Point, 46

Northern, 61

Southwestern, 46, 120, 122, 389, 407

Orthoptera, 319

Orthopteroid, 319

Ouellet, M., 378

Ovenden, L. Minutes of the $139^{\text {th }}$ Annual Business Meeting (ABM) of the Ottawa Field-Naturalists' Club 9 January 2018, 87-88

Oviposition, 103

Park,

Algonquin Provincial, 20, 58, 103

Rondeau Provincial, 122

Waterton Lakes National

Parrett, L.S., 382

Parturition, 219

Paszkowski, C.A., 168

Patterns, Activity, 20

Peeper, Spring, 43, 163

Peller, P., 149

Permafrost, 279

Petromyzon marinus, 120

Phenology, 219

Pilgrim, K.L., 268

Pine, Whitebark, 285

Pinus albicaulis, 285

Pituophis catenifer

deserticola, 30

sayi, 126,140

Plant, 231, 286 
Plethodon cinereus, 58

Poecile atricapillus, 368

Pollard, W.H., 279

Polycyclic Aromatic Hydrocarbon, 103

Population, 20 Breeding, 53

Poulin, R.G., 108, 126

Powell, G.L., P. Peller, A.P. Russell. Incidentally gathered natural history information on Bullsnakes (Pituophis catenifer sayi) in southeastern Alberta, 140-149

Powell, T., 219

Power, T., 4

Power, T., J. Gilhen. Status, distribution, and nesting ecology of Snapping Turtle (Chelydra serpenti$n a)$ on Cape Breton Island, Nova Scotia, Canada, $8-17$

Prairies, Tallgrass, 407

Predation, 103, 122, 382

Predator, 122, 223

Prey, 223

Price, C.J., 168

Prince Edward Island, 330

Procyon lotor, 122

Pseudacris crucifer, 43

Pteridophyte, 350

Quebec,

Frontenac Axis, 350

Southern, 368, 378

Western, 25, 264

Raccoon, 122

Racer, Western Yellow-bellied, 30

Railway, 389

Range, 4, 163

Home, 126

Range Extension, 4, 163, 231, 254, 261, 264, 319, 350,389

Rangifer tarandus, 382

Rashleigh, K.R., M. Crowell. Spring Peeper (Pseudacris crucifer) in Labrador, Canada: an update, 163167

Rate, Growth, 25

Ratio, Sex, 289

Rattlesnake, Northern Pacific, 30

rDNA, 407

Record(s),

Breeding, 254

First, 261

Size, 378

Refugia, Overwintering, 168

Rehabilitation, Wildlife, 289

Reproduction, 150, 219 Lifelong, 211

Reptile, 4, 8, 18, 20, 25, 30, 36, 103, 108, 120, 122, $126,140,150,176,223,289,378$

Richness,

Diet, 268

Species, 176
River,

Mira, 4, 8

Saint John, 231

Rivière du Sud, 378

Robards, M.D., 268, 382

Robinson, S.L, 319

Russell, A.P., 140

Rutherford, P.L., 150

Saarela, J.M., 85

Sadar, M.J., 126

Salamander,

Eastern Red-backed, 58

Long-toed, 168

Mudpuppy, 61

Sampling, Soil rDNA, 407

Saskatchewan,

Regina, 108

Scat, 268

Southern, 126

Science, Citizen, 330, 394, 434

Scrimgeour, G.J., 168

Seasonality, 20

Seburn, D.C., 1

Seburn, D. Record longevity of a Spotted Turtle (Clemmys guttata), 18-19

Seburn, D.C., W.D. Halliday. The publications of Francis Cook, $99-102$

Selection,

Habitat, 46, 108, 126, 223

Mate, 211

Sequencing, Next-generation, 407

Sex Ratio, 20

Sivy, K.J., 268

Size,

Body, 36

Clutch, 8

Litter, 36

Skate, Commander, 261

Skuza, J., 120

Slough, B.G., A. DeBruyn. The observed decline of Western Toads (Anaxyrus boreas) over several decades at a novel winter breeding site, 53-57

Slug, 264

Slump, Thaw, 279

Snake,

Bullsnake, 126, 140

Common Gartersnake, 25, 223

Grassland, 126

Great Basin Gophersnake, 30

Northern Pacific Rattlesnake, 30

Red-bellied, 150

Western Gartersnake, 36

Western Yellow-bellied Racer, 30

Softshell, Spiny, 120

Somers, C.M., 108, 126

Species,

New, 319

Rare, 394

Spiders, 330 
Spread, 264

St. John's-wort, Round-fruited, 389

Stores, Fat, 368

Structure,

Habitat, 223

Vegetation, 223

Species, Endangered, 176

Springs, Warm, 53

Storeria occipitomaculata, 150

Success, Hatching, 8

Survey, 407

Swift, White-throated, 386

Swimming, 386

Taxonomy, 360

Telemetry,

Passive Integrated Transponder, 168 Radio, 46, 108, 126

Temperate, 150

Temperature, 150, 368 Body, 25

Temporal, 368

Territoriality, 58

Thamnophis elegans, 36

sirtalis, 25

Thermoregulation, 25, 150

Thorn, R.G., 407

Toad,

Fowler's, 46

Western, 53

Turtle, 289

Blanding's, 122, 289

Northern Map, 122, 289

Painted, 20, 108, 289

Snapping, 4, 8, 103, 122, 289, 378

Spiny Softshell, 120

Spotted, 18

Western Painted, 108
Twardek, W.M., 61

Use,

Habitat, 46, 58, 108, 126, 140, 150, 168

Landscape, 140

Space, 126

Valkenburg, P., 382

Van Hemessen, W.D., 389

Vanderwel, M.C., 126

Variation, Colour, 43

Vegetation, 223, 231, 238

Vocalization, 163

Water-milfoil, Eurasian, 231

Watershed,

Colville River, 268

Mira River, 4

Wascana Creek, 108

Wetland, 223

Whitear, A.K., 120

Wildlife, 279

Wolverine, 268, 382

Wormslug, 264

Yagi, K.T., 46

Yukon,

Herschel Island, 279

Southwestern, 219

Zubkowksi, T.B., 261

Zurbrigg, E., I. Brodo, J. Cipriani, C. Hanrahan, K. McLachlan Hamilton. The Ottawa Field-Naturalists' Club Awards for 2017, presented February 2018, 95-98. 


\section{Index to Book Reviews}

\section{Botany}

Bickerton, H. "Catalogue of the Vascular Plants of New York State, Memoirs of the Torrey Botanical Society, Volume 27" by David Werier, 2017, 67-68

Bocking, E. "Carnivorous Plants: Physiology, Ecology, and Evolution" by Aaron M. Elison and Lubomír Adamec, 2018, 191-192

Brunton, D.F. "Flora of Florida Volume 5 (Dicotyledons, Gisekiaceae through Boraginaceae)" by R.P. Wunderlin, B.F. Hansen, and A.R. Franck, 2018, 68

Brunton, D.F. "Islands of Grass" by Trevor Herriot, photography by Branimir Gjetvaj, 2017, 69

Crins, W.J. "Sedges and Rushes of Minnesota: The Complete Guide to Species Identification" by Welby R. Smith, photography by Richard Haug, 2018, 296-297

Iles, M. "Woody Plants of the Northern Forest - A Photographic Guide" by Jerry Jenkins, 2018, 297298

Iles, M. "Woody Plants of the Northern Forest - Quick Guide" by Jerry Jenkins, 2018, 297-298

\section{Entomology}

Catling, P. "Lady Beetles of the Northwest Territories" by Environment and Natural Resources, 2018, 70

Catling, P. "Naïades et exuvies des libellules du Quebec : clé de determination des genres" by Raymond Hutchinson and Benoit Ménard, 2016, $71-72$

Halliday, W.D. "A Field Guide to Insects of the Pacific Northwest" by Robert Cannings, 2018, 73

Lauff, R. "Beetles: The Natural History and Diversity of Coleoptera" by Stephen A. Marshall, 2018, 193

Marshall, S.A. "Amazing Arachnids" by Jillian Cowles, 2018, 192-193

Smith, T. "The Green Menace: Emerald Ash Borer and the Invasive Species Problem" by Jordan D. Marché II, 2017, 194-195

\section{Herpetology}

Seburn, D. "Ecology and Conservation of the Diamond-backed Terrapin" Edited by W.M. Roosenburg and V.S. Kennedy, 2019, 425

\section{Ornithology}

Armstrong, T. "The Birds at My Table: Why We Feed Wild Birds and Why It Matters" by Darryl Jones, 2018, 301-302

Cannings, S. "The Birds of Vancouver Island's West Coast" by Adrian Dorst, 2018, 298-299

Cray, H.A. "The Genius of Birds" by Jennifer Ackerman, 2016, 426
Crins, W.J. "Seabird Colonies of British Columbia: A Century of Changes" by Michael S. Rodway, R. Wayne Campbell, and Moira J.F. Lemon, 2017, 195-196

John, R. "Best Places to Bird in Ontario" by Kenneth Burrell and Michael Burrell, 2019, 426-427

Montevecchi, B. "The Birds of Nunavut, Volume 1: Nonpasserines, Volume 2: Passerines" by James M. Richards and Anthony J. Gaston, 2018, 197198

Smith, C.M. "North American Ducks, Geese \& Swans Identification Guide" by Frank S. Todd, 2018, 196-197

Smith, C.M. "The Ascent of Birds: How Modern Science is Revealing Their Story" by John Reilly, 2018, 299-300

Smith, C.M. "The Cooper's Hawk: Breeding Ecology \& Natural History of a Winged Huntsman" by Robert N. Rosenfield, 2018, 300-301

\section{Other}

Beaudoin, A.B. "Curators: Behind the Scenes of Natural History Museums" by Lance Grande, 2017, 307-309

Cottam, B. New Titles, 82-84, 204-208, 311-313, 430432

Cottam, B. "Through a Glass Brightly: Using Science to See Our Species as We Really Are" by David B. Barash, 2018, 202-203

Gaston, T. "The Inner Life of Animals: Love, Grief and Compassion - Surprising Observations of Hidden World" by Peter Wohlleben, 2017, 76

Gaston, T. "The Subjugation of Canadian Wildlife: Failures of Principle and Policy" by Max Foran, 2018, 77

Halliday, W.D. "Half-Earth: Our Planet's Fight for Life" by Edward O. Wilson, 2017, 78

Houston, C.S. "Mark Catesby's Legacy: Natural History Then and Now" by M.J. Brush and Alan H. Brush, 2018, 203-204

Smith, C.M. "Best Places to Bird in the Prairies" by John Acorn, Alan Smith, and Nicola Koper, 2018, 79

Tegler, B. "The Marsh Builders: The Fight for Clean Water, Wetlands, and Wildlife" by Sharon Levy, 2018, 309-310

Way, J. “Deep Into Yellowstone: A Year's Immersion in Grandeur and Controversy" by Rick Lamplugh, 2017, 80-81

\section{Zoology}

Catling, P. "A Natural History Study of Leech (Annelida: Clitellata: Hirudinida) Distributions in Western North America North of Mexico?" by Peter Hovingh, 2016, 74-75 
Cray, H.A. "Eye of the Shoal" by Helen Scales, 2018, 303

Cray, H.A. "Spineless: The Science of Jellyfish and the Art of Growing a Backbone" by Juli Berwald, 2018, 304

Cray, H.A. "Immersion: The Science and Mystery of Freshwater Mussels" by Abbie Gascho Landis, 2017, 306-307

Halliday, W.D. "Wildlife of the Arctic" by Richard Sale and Per Michelsen, 2018, 198-199

Halliday, W.D. "Spying on Whales" by Nick Pyenson, 2018, 429
Twardek, W.M. "Guide to the Parasites of Fishes of Canada Part V: Nematoda" by Hisao P. Arai and John W. Smith, 2016, 199-200

Wachelka, H. "Muskellunge Management: Fifty Years of Cooperation Among Anglers, Scientists, and Fisheries Biologists" by Kevin Kapuscinski, Timothy Simonson, Derek Crane, Steven Kerr, James Diana, and John Farrell, 2017, 427-428

Way, J. "Mountain Lions of the Black Hills: History and Ecology" by Jonathan A. Jenks, 2018, 200201

Way, J. "Keepers of Wolves. Second Edition" by Richard P. Thiel, 2018, 305-306 